\title{
Low-energy neutrino-nucleus interactions and beta-beam neutrino
}

\author{
N.Jachowicz, V. Pandey \\ Ghent University, Department of Physics and Astronomy, Proeftuinstraat 86, B-9000 Gent, Belgium.
}

\begin{abstract}
We present an overview of neutrino-nucleus scattering at low energies with cross sections obtained within a continuum random phase approximation (CRPA) formalism. We highlight potential applications of beta-beam neutrino experiments for neutrino astrophysics. Our calculations are compared with MiniBooNe data at intermediate energies.
\end{abstract}

Keywords: neutrino-nucleus interactions, long-range correlations, beta-beams

PACS: $25.30 . P t, 24.10 . J v, 24.70,+s, 14.20 . \mathrm{Dh}$

\section{INTRODUCTION}

Most current experimental efforts focus on neutrinos with energies of a few hundreds of MeVs up to energies in the $\mathrm{GeV}$ range. Still, neutrinos are produced in a variety of phenomena and at various sites in the universe and hence come in different flavours and with a broad range of energies, with a lot of neutrinos having energies below $100 \mathrm{MeV}$. Learning from these neutrino involves the need to detect them in their interactions with matter. Neutrino-electron scattering cross sections are theoretically well-known, but have the disadvantage that the cross sections are very small. Scattering off nuclei results in larger cross sections. At small incoming neutrino energies, the interaction process is however very sensitive to details in nuclear structure and to the influence of correlations between the nucleons.

\section{MODELING NEUTRINO-NUCLEUS INTERACTIONS AT LOW ENERGIES}

The study of the atomic nucleus faces particular problems : generally, the atomic nucleus is a mesoscopic system, on the one hand containing too many particles to allow few-body techniques to be effective and on the other hand containing too few nucleons to enable a statistical approach of the problem. In the tens-of-MeV energy range important for e.g. supernova neutrinos, cross sections are very sensitive to nuclear structure effects.

The main methods to study neutrino scattering off nuclei at supernova-neutrino energies are the Shell Model (SM) and the Random Phase Approximation (RPA). In the former, the description of the nucleus is based on a full diagonalization of a effective interaction in a limited model space. In recent years, the shell model has been used successfully to study various weak interactions of interest to nuclear astrophysics [1]. The main disadvantage of the shell model approach is the dimension of the matrices to be diagonalized, rapidly growing with increasing model sizes.

Confronted with this drawback, a number of approximations has been designed, focusing on various aspects of the problem. Next to the Hartree-Fock approximation, considering only single-particle properties of the problem, more elaborate techniques as e.g. the RPA were developed. Contrary to mean-field descriptions where a nucleon experiences the presence of the others only through the mean-field generated by their mutual interactions, the random phase approximation allows correlations to be present even in the ground state of the nuclear system and additionally allows the particles to interact by means of the residual two-body force. The random phase approximation then describes a nuclear state as the coherent superposition of particle-hole contributions out of a correlated ground state :

$$
\left|\Psi_{R P A}\right\rangle=\sum_{c}\left\{X_{(\Psi, C)}\left|p h^{-1}\right\rangle-Y_{(\Psi, C)}\left|h p^{-1}\right\rangle\right\} .
$$

The summation index $C$ stands for all quantum numbers defining a reaction channel unambiguously :

$$
C=\left\{n_{h}, l_{h}, j_{h}, m_{h}, \varepsilon_{h} ; l_{p}, j_{p}, m_{p}, \tau_{z}\right\}
$$

where the indices $p$ and $h$ indicate whether the considered quantum numbers relate to the particle or the hole state, $\varepsilon_{h}$ denotes the binding-energy of the hole state and $\tau_{z}$ defines the isospin character of the particle-hole pair. In standard 
RPA calculations this leads to a discrete spectrum, with several variations in the approach [2, 3, 4] in use. In this contribution, the cross section results are illustrated using a Continuum Random Phase Approximation (CRPA), based on a Green's function approach $[5,6,7]$. The unperturbed wave-functions are generated using either a Woods-Saxon potential or a HF-calculation using a Skyrme force. The latter approach makes self-consistent HF-RPA calculations possible.

The differential cross-section for scattering of an incoming neutrino with energy $\varepsilon_{i}$ of a nucleus is given by

$$
\left(\frac{d^{2} \sigma_{i \rightarrow f}}{d \Omega d \omega}\right)_{\bar{v}}=\frac{G^{2} \varepsilon_{f}^{2}}{\pi} \frac{2 \cos ^{2}\left(\frac{\theta}{2}\right)}{2 J_{i}+1}\left[\sum_{J=0}^{\infty} \sigma_{C L}^{J}+\sum_{J=1}^{\infty} \sigma_{T}^{J}\right],
$$

with

$$
\begin{aligned}
& \sigma_{C L}^{J}=\left|\left\langle J_{f} \| \widehat{\mathscr{M}_{J}}(\kappa)+\frac{\omega}{|\vec{q}|} \widehat{\mathscr{L}_{J}}(\kappa)|| J_{i}\right\rangle\right|^{2}, \\
& \sigma_{T}^{J}=\left(-\frac{q_{\mu}^{2}}{2|\vec{q}|^{2}}+\tan ^{2}\left(\frac{\theta}{2}\right)\right)\left[\left|\left\langle J_{f} \| \widehat{\mathscr{J}}_{J}^{\text {mag }}(\kappa)|| J_{i}\right\rangle\right|^{2}+\left|\left\langle J_{f} \| \widehat{\mathscr{J}}_{J}^{l}(\kappa)|| J_{i}\right\rangle\right|^{2}\right] \\
& \mp \tan \left(\frac{\theta}{2}\right) \sqrt{-\frac{q_{\mu}^{2}}{|\vec{q}|^{2}}+\tan ^{2}\left(\frac{\theta}{2}\right)}\left[2 \Re\left(\left\langle J_{f} \| \widehat{\mathscr{J}}_{J}^{\text {mag }}(\kappa)|| J_{i}\right\rangle\left\langle J_{f}\left\|\widehat{\mathscr{J}}_{J}^{e l}(\kappa)\right\| J_{i}\right\rangle^{*}\right)\right],
\end{aligned}
$$

where $\widehat{\mathscr{M}}_{J}$ and $\widehat{\mathscr{L}}_{J}$ are the Coulomb and longitudinal multipole operators, $\widehat{\mathscr{J}}_{J}^{\text {mag }}$ and $\widehat{\mathscr{J}}_{J}^{e l}$ the transverse multipole operators. $\theta$ is the scattering angle of the lepton. For each multipole transition $J^{\pi}$ only one part -vector or axial vectorof an operator is contributing. From the expression (3) it is clear that $\mathrm{J}=0$ transitions are suppressed due to the lack of a transverse contribution in these channels. Still, neutrinos are able to excite $0^{-}$states in nuclei, while electrons cannot. The second and third part of the expression show that there is interference between the Coulomb and the longitudinal (CL) terms and between both transverse contributions, but not between transverse and CL terms. The only difference between neutrino and antineutrino cross-sections is in the opposite sign of the transverse interference part. From the angular dependence of the kinematic factors, it is clear that for backward $\theta=\pi$ scattering only transverse terms contribute, while for $\theta=0 \mathrm{CL}$-contributions dominate.

\section{CROSS SECTION RESULTS}

Neutrino scattering potentially constitutes a rich source of information on nuclear structure and weak interaction characteristics. But notwithstanding the experimental efforts, the extraction of information out of scattering reactions is very difficult, due to the very small interaction cross-sections. The importance of neutrinos in a variety of astrophysical situations therefore represents an important additional motivation for the study of neutrino-nucleus scattering reactions.

In this section, the main characteristics of neutrino-nucleus scattering at supernova-neutrino energies are discussed using the example of neutral-current scattering of $50 \mathrm{MeV}$ neutrinos off ${ }^{16} \mathrm{O}$.

The right panel of figure 1 shows the differential cross-section for this reaction as a function of the excitation energy $\omega$ of the nucleus, and its most important multipole contributions. The differential neutrino scattering crosssections are of the order of $10^{-42} \mathrm{~cm}^{2}$ per MeV. The figure clearly illustrates that at energies below $20 \mathrm{MeV}$, the cross-section spectrum is sharply peaked. These peaks are related to excitations with a strong single-particle character. The resonances are however very narrow and therefore do not absorb all transition strength. At excitation energies between 20 and $25 \mathrm{MeV}$, the broad resonance structure of the giant dipole resonance shows up. For excitation energies above approximately $30 \mathrm{MeV}$ the cross-section decreases almost purely quadratically as a function of the excitation energy of the nuclear system. This agrees with the energy dependence of equation (3) which shows the cross-section to be proportional to the square of the outgoing lepton energy $\frac{d \sigma}{d \omega} \sim\left(\varepsilon_{f}\right)^{2}$. This effect furthermore results in the smooth and soft broadening of the resonances for higher values of the energy of the lepton projectile.

In the calculation, multipoles up to $\mathrm{J}=4$ were taken into account. Contributions of higher-order multipole excitations were found to be very small at the considered energies. The $\mathrm{J}=5$ transitions are suppressed by almost 5 orders of magnitude and as a consequence have negligible influence on the total cross-section. The smooth behavior of these contributions furthermore assures that the shape of the resonance structure in the excitation spectrum is not 

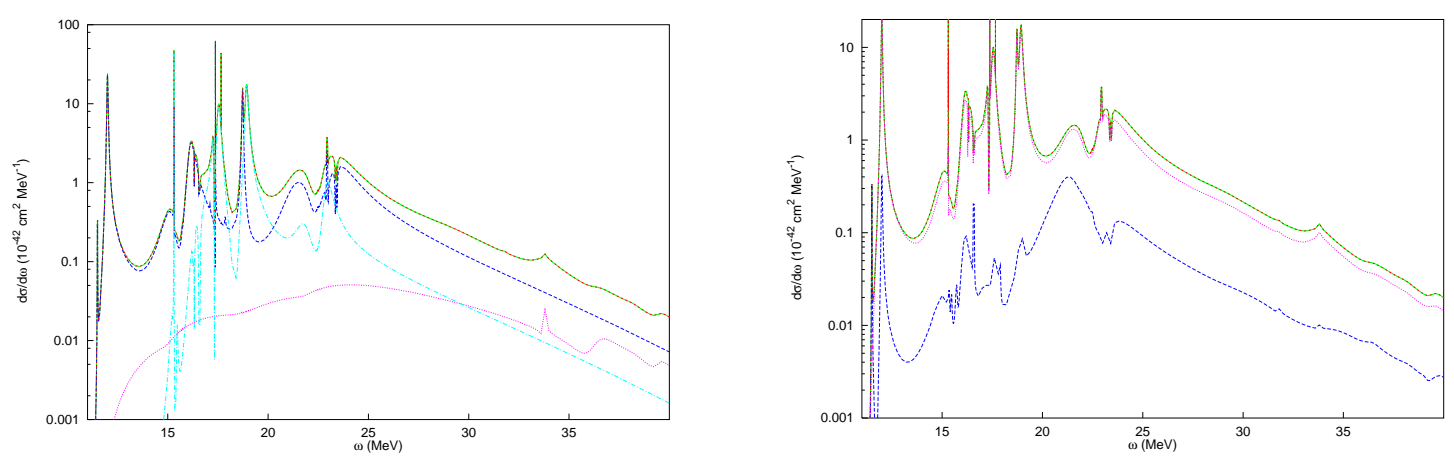

FIGURE 1. Left : Cross-section for the neutral current reaction ${ }^{16} O+v_{50 \mathrm{MeV}} \rightarrow{ }^{16} \mathrm{O}^{*}+v^{\prime}$ (full line) and its dominant multipole contributions. $J^{\pi}=1^{-}$(dashed line), $J^{\pi}=1^{+}$(dashed-dotted) and $J^{\pi}=2^{-}$(dotted line). The total cross-section includes multipoles up to J=4. Right : Comparison between the vector (dashed) and the axial vector (dotted line) contribution to the reaction ${ }^{16} O\left(v, v^{\prime}\right){ }^{16} O^{*}$. In both figures, the single-particle wave-functions and energies were obtained with a Hartree-Fock calculation, as residual interaction the SkE2 parameterization was used.

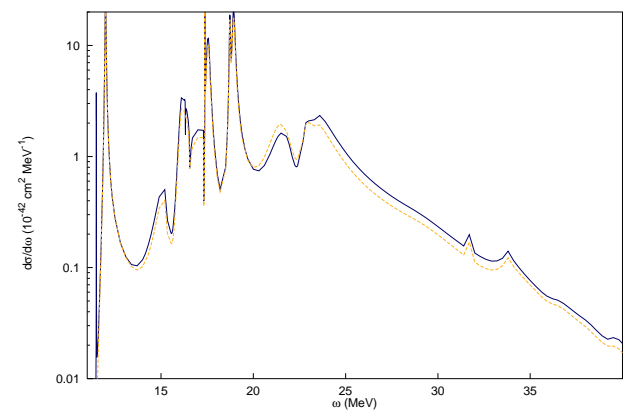

FIGURE 2. Cross-section for neutral current neutrino (full line) and antineutrino (dashed) scattering reactions off oxygen 16.

affected by the higher-order multipole transitions. The $\mathrm{J}=1$ excitations are prominent, with a clear dominance of the $\mathrm{J}=1^{-}$electric dipole transition in the giant resonance region. Next to higher-order multipole transitions $\mathrm{J}=3$ and $\mathrm{J}=4$, also $\mathrm{J}=0$ excitations are suppressed. This is due to the fact that only Coulomb and longitudinal terms contribute to these channels. But still, some clear $0^{-}$resonances show up in the differential cross-sections. In general, negative parity transitions are clearly dominating the positive parity contributions. For higher excitation energies, the relative importance of higher-order multipoles increases. The right panel of the figure shows that the axial contribution is clearly dominant.

Figure 2 compares neutrino- and antineutrino-induced cross sections. Whereas differences are small at these energies, neutrino cross sections are larger. This difference will grow at higher energies with the increasing importance of the transverse magnetic contribution.

\section{LOW-ENERGY BETA-BEAM NEUTRINOS}

Beta beams, which are neutrino beams produced by the beta decay of nuclei that have been accelerated to high gamma factor, were originally proposed for high energy applications [8], such as the measurement of the third neutrino mixing angle $\theta_{13}$ [8]. Refs. [9, 10] suggested that a beta beam run at lower gamma factor, would be useful for neutrino measurements at lower energies.

We suggested $[11,12]$ exploiting the flexibility these beta-beam facilities offer, combined with the fact that betabeam neutrino energies overlap with supernova-neutrino energies, to construct 'synthetic' spectra that approximate an incoming supernova-neutrino energy-distribution [13]. Using these constructed spectra we are able to reproduce total and differential folded supernova-neutrino cross-sections very accurately. We construct linear combinations of beta-beam energy-distributions

$$
n_{N \gamma}\left(\varepsilon_{i}\right)=\sum_{i=1}^{N} a_{i} n_{\gamma_{i}}\left(\varepsilon_{i}\right) \text {, }
$$



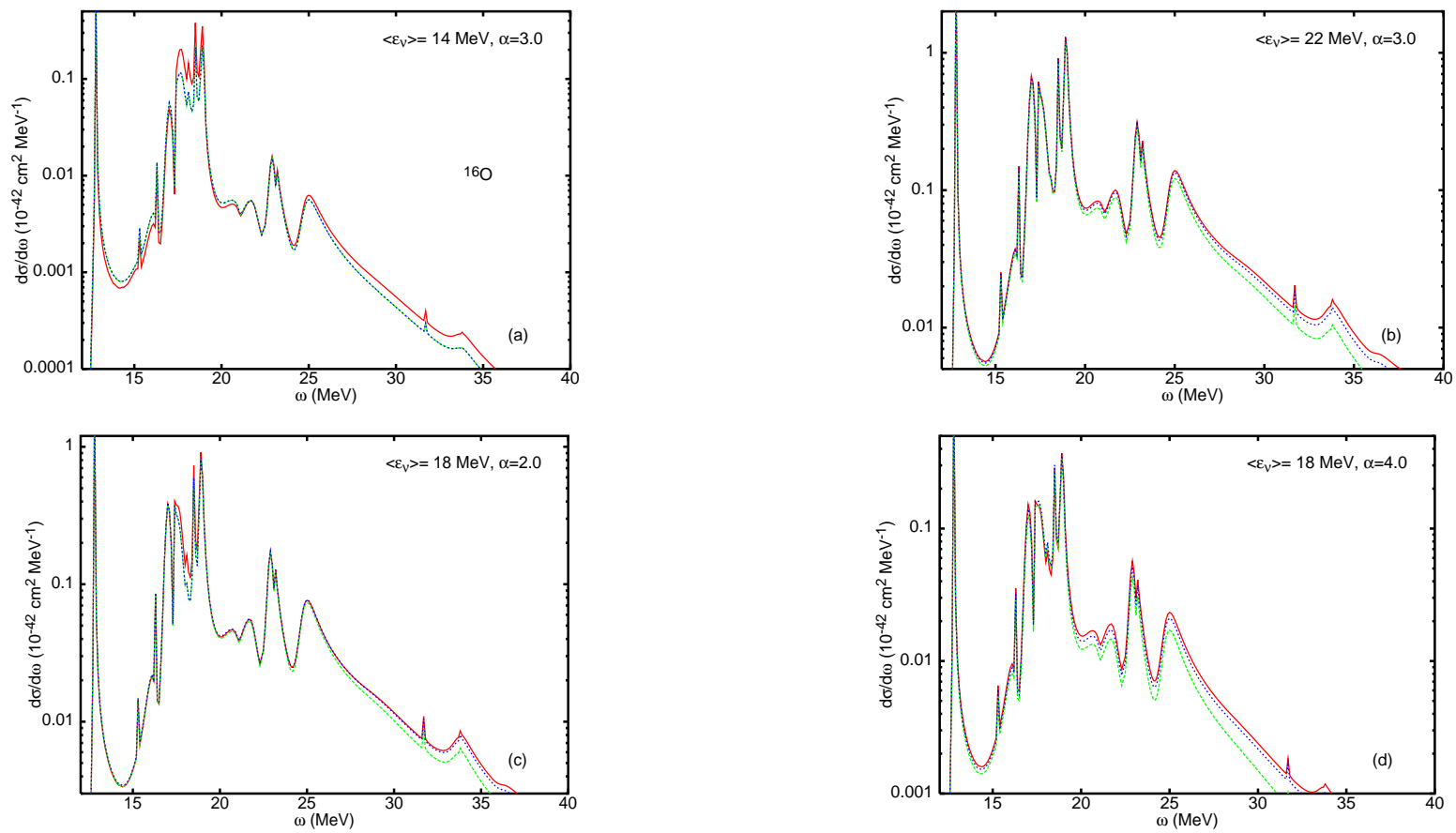

FIGURE 3. Comparison between differential cross sections for neutral-current scattering on ${ }^{16} \mathrm{O}$, folded with a power-law supernova-neutrino spectrum (full line) and synthetic spectra with 3 (dashed line) and 5 components (dotted line) for different energy distributions : $\langle\varepsilon\rangle=14, \alpha=3$ (a), $\langle\varepsilon\rangle=22, \alpha=3$ (b), $\langle\varepsilon\rangle=18, \alpha=2$ (c), and $\langle\varepsilon\rangle=18, \alpha=4$ (d).

where all distributions involved were normalized to 1 :

$$
\int d \varepsilon_{i} n_{N \gamma}\left(\varepsilon_{i}\right)=1
$$

and

$$
\int d \varepsilon_{i} n_{\gamma_{i}}\left(\varepsilon_{i}\right)=1 \quad \forall i
$$

The constructed spectrum that represents the best fit to the supernova-spectrum is then obtained by minimizing the expression

$$
\int_{\varepsilon_{i}} d \varepsilon_{i}\left|n_{N \gamma}\left(\varepsilon_{i}\right)-n_{S N}\left(\varepsilon_{i}\right)\right|
$$

where the similarities between beta-beam and supernova neutrino spectra assure that a good fit is easily obtained. In this way, the values for the expansion parameters $a_{i}$ and the boost factors $\gamma_{i}$ that yield a spectrum that is as close as possible to the original power-law distribution are determined. Count rate considerations favor higher gammas, for example $\gamma$-values between 5 and 15 .

Of course the spectrum as such is not an important observable. The information brought along by supernova neutrinos is encoded in the response of the detector to the incoming neutrino flux. This quantity is determined by the folded differential cross section : the folded cross section as a function of the excitation energy of the target indicates what the neutrino signal in the detector will look like. In fig. 3, we show the differential cross section for neutral-current neutrino scattering off an ${ }^{16} \mathrm{O}$ target for different energy distributions. The agreement between cross sections folded with the power-law supernova-neutrino spectrum and those folded with the synthetic spectrum is remarkably good. The procedure is able to reproduce total strength, and the position and width of the resonances very accurately. This result suggests that beta-beam neutrino-scattering measurements can be a very effective way of predicting supernovaneutrino responses without need to go through to the intermediate step of a cross section calculation with the related uncertainties. In principal, the formalism can be applied to any target material. Its efficiency however depends on the accuracy of the fitting procedure in the relevant energy range. 


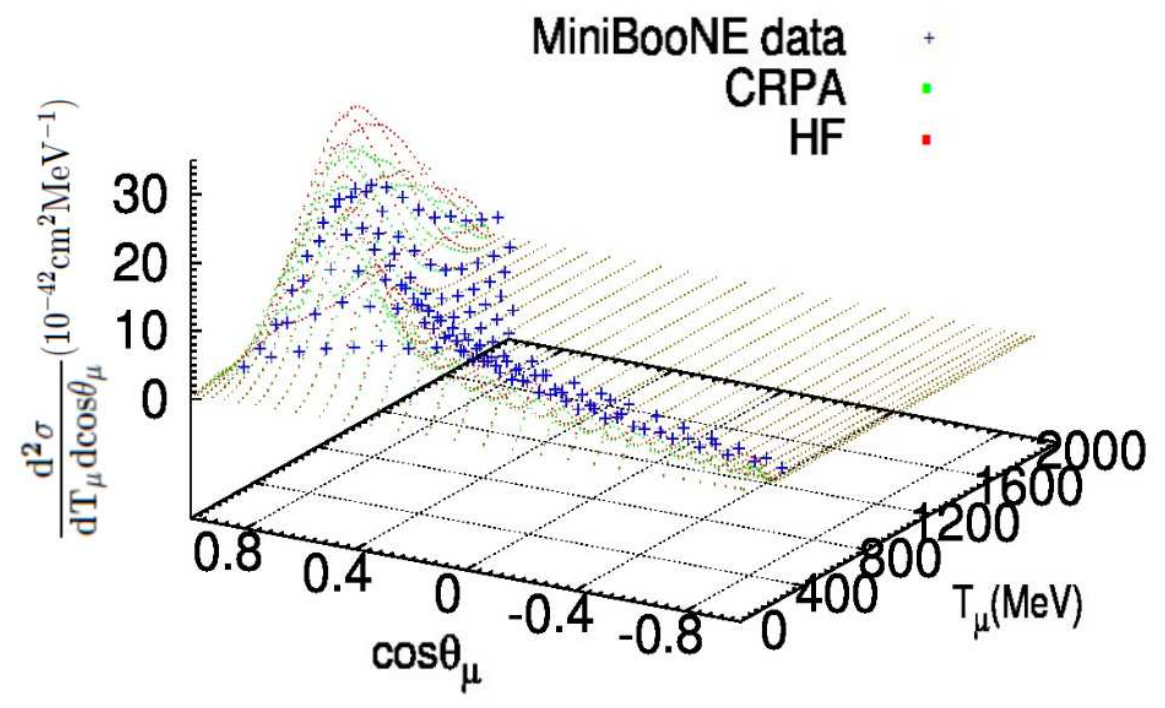

FIGURE 4. Comparison between our CRPA calculations and MiniBooNe data. The cross section is shown as a function of the lepton scattering angle and the muon energy. In the calculaltions, all multipoles up to $\mathrm{J}=12$ were included.

\section{COMPARISON WITH EXPERIMENTAL DATA AT HIGHER ENERGIES}

At higher incoming energies, our results can be compared with the MiniBooNe data [14]. Figure shows double differential cross sections for charged current neutrino scattering off a ${ }^{12} \mathrm{C}$ target nucleus, folded with the MiniBooNe spectrum. The overall behavior of the cross section is well reproduced. The figure compares Hartree-Fock and CRPA results, clearly indicating that the inclusion of long-range correlations is mandatory for a good description of the data. Further work in this direction is in progress [15].

\section{ACKNOWLEDGMENTS}

This research was funded by the Interuniversity Attraction Poles Programme initiated by the Belgian Science Policy Office and by the Erasmus Mundus External Cooperations Window's Eurindia Project.

\section{REFERENCES}

1. K. Langanke, G. Martínez-Pinedo, B. Müller, H.-Th. Janka et al, Phys. Rev. Lett 100, 011101 (2008).

2. E. Kolbe, K. Langanke, S. Krewald and F.K. Thielemann, Nucl. PhysA540, 599 (1992).

3. C. Volpe, N. Auerbach, G. Coló, T. Suzuki, N. Van Giai, Phys. Rev. C 62, 015501 (2000).

4. A. Samana, F. Krmpotić, N. Paar, C. Bertulani, Phys. Rev. C 83, 024303 (2011).

5. N. Jachowicz, K. Heyde, J.Ryckebusch, and S. Rombouts, Phys. Rev. C59,3246 (1999).

6. N. Jachowicz, K. Heyde, J.Ryckebusch, and S. Rombouts, Phys. Rev. C65, 025501 (2002).

7. N. Jachowicz, K. Heyde, and J. Ryckebusch, Phys. Rev. C 66, 055501 (2002).

8. P. Zuchelli, Phys. Lett. B532, 166 (2002).

9. C. Volpe, J. Phys. G30, 1 (2004).

10. G. C. McLaughlin, Phys. Rev. C 70, 045804 (2004).

11. N. Jachowicz, G.C. McLaughlin, Phys. Rev. Lett. 96, 172301 (2006).

12. N. Jachowicz, G.C. McLaughlin, C. Volpe, Phys. Rev. C77, 055501 (2008).

13. N. Jachowicz, K. Heyde, Phys. Rev. C 68, 055502 (2003).

14. The MiniBooNe collaboration, Phys. Rev. D 81, 092005 (2010.

15. V. Pandey, N. Jachowicz, J. Ryckebusch, in preparation. 